\title{
NMR Imaging of Low Pressure, Gas-Phase Transport in Packed Beds Using Hyperpolarized Xenon-129
}

\author{
Galina Pavlovskaya, Joseph Six, and Thomas Meersman \\ Sir Peter Mansfield Imaging Centre, School of Medicine, University of Nottingham, University Park, Nottingham \\ NG7 2RD, U.K. \\ Navin Gopinathan and Sean P. Rigby \\ Dept. of Chemical and Environmental Engineering, University of Nottingham, University Park, Nottingham \\ NG7 2RD, U.K.
}

\author{
DOI 10.1002/aic.14929 \\ Published online in Wiley Online Library (wileyonlinelibrary.com)
}

\begin{abstract}
Gas-phase magnetic resonance imaging (MRI) has been used to investigate heterogeneity in mass transport in a packed bed of commercial, alumina, catalyst supports. Hyperpolarized ${ }^{129}$ Xe MRI enables study of transient diffusion for microscopic porous systems using xenon chemical shift to selectively image gas within the pores, and, thence, permits study of low-density, gas-phase mass-transport, such that diffusion can be studied in the Knudsen regime, and not just the molecular regime, which is the limitation with other current techniques. Knudsen-regime diffusion is common in many industrial, catalytic processes. Significantly, larger spatial variability in mass transport rates across the packed bed was found compared to techniques using only molecular diffusion. It has thus been found that that these heterogeneities arise over length-scales much larger than $\sim 100 \mu \mathrm{m}$. (C) 2015 American Institute of Chemical Engineers AIChE J, 00: 000000, 2015
\end{abstract}

Keywords: gas-phase magnetic resonance imaging, hyperpolarized xenon, Knudsen diffusion, catalyst support

\section{Introduction}

Heterogeneous catalysts, such as metals, are usually dispersed on a (relatively) inert, porous support pellet with both high surface area and large overall size, to maximize active surface area, but also to reduce reactor pressure-drop. ${ }^{1}$ While a large support pellet with small pores will have a large active surface area, these characteristics will also reduce the rate of diffusion, often causing mass transport to be the rate-limiting step rather than reaction rate. Further, the concentration gradients of different species arising from the diffusion limitation can alter the balance between competing reactions and change catalyst selectivity. A common design $^{2}$ employed to attempt to resolve this issue is to use pellets with a bimodal pore-size distribution consisting of a pervasive macroporous network, to provide rapid access to the interior of the pellet, with mesopores branching to provide extensive surface area for the catalyst. The issues that arise with this design are whether the macroporous network is effective in improving access to the center of the pellets, and whether the diffusive performance of pellets is uniform.

Although it is possible to design catalyst supports with well-defined and optimized pore geometry for a given reaction, ${ }^{3}$ the currently used support materials for this purpose are prone to structural evolution and collapse under the hydrothermal conditions frequently encountered in many industrial processes. Hence, most industrial catalysts are still supported

\footnotetext{
Correspondence concerning this article should be addressed to S. P. Rigby at enzspr@exmail.nottingham.ac.uk.
}

(C) 2015 American Institute of Chemical Engineers on amorphous materials with a more complex pore space geometry. This raises the question of whether all pellets have similar mass transport performance. Bed heterogeneity is an issue when trying to predict the overall performance of a reactor. There is thus a need for a method to test for these issues. Further, the precise and accurate structural characterization of these materials remains controversial, and, therefore, is an area of on-going study. It is thus difficult to construct sufficiently accurate structural models of amorphous materials on which to conduct a priori predictive simulations of diffusion and reaction. Therefore, experimental studies of mass transport properties offer an alternative way to assess the likely properties of different support pellets. In turn, the experimental data will also assist in the refinement of simulation programs. The technique presented in this article provides a method to check the level of heterogeneity at the pellet and bed scale, which will be demonstrated for a case study of a commercial bimodal alumina tablet.

A number of different experimental techniques have been used to study mass transport in catalyst support pellets. These tend to fall into either steady-state or transient methods. Steady-state methods include the Wicke-Kallenbach cell ${ }^{4}$ and pulsed-field gradient (PFG) nuclear magnetic resonance (NMR). ${ }^{5}$ Transient methods include adsorption-uptake, ${ }^{6}$ scattering methods, ${ }^{7}$ inverse gas chromatography, ${ }^{8}$ and fluid-fluid exchange experiments. ${ }^{9}$ Transient methods are often preferred because they more closely mimic the dynamic processes in catalytic reactors. Steady-state methods, especially throughflow methods like the Wicke-Kallenbach cell, are more 

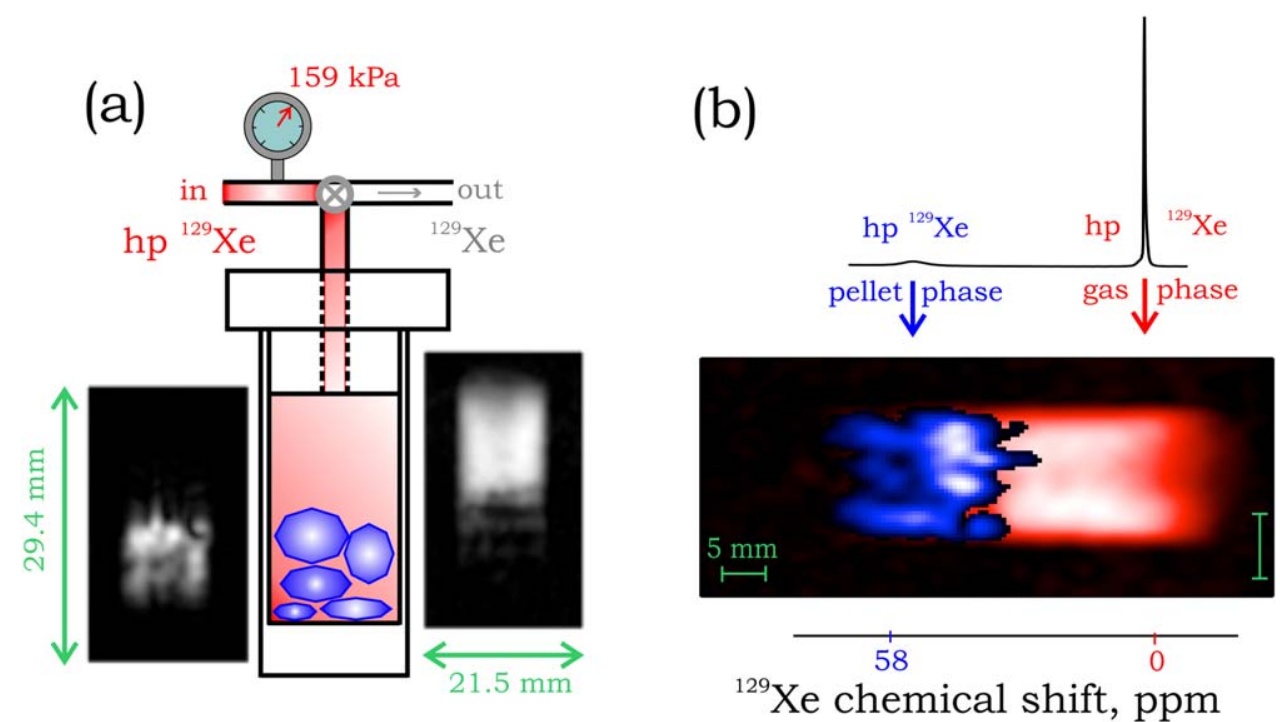

Figure 1. (a) The outline of the pellet sample holder. The sample container is evacuated and hp ${ }^{129} \mathrm{Xe}$ is delivered through the valve as shown. Pressure in the sample container is equalized to $159 \mathrm{kPa}$ after the gas delivery. The images shown depict $\mathrm{hp}{ }^{129} \mathrm{Xe}$ in the pellet phase (left) and in the gas phase (right); (b) ${ }^{129} \mathrm{Xe} \mathrm{1-D}$ spectrum of xenon gas in the pellet sample. The chemical shift separation between gas and the pellet phase is $58 \mathrm{ppm}$. A composite image combined from the images acquired at the pellet phase frequency (blue) and at the gas-phase frequency (red) is also shown. Please note the match in image boundaries at the interface between the gas and pellet phases.

[Color figure can be viewed in the online issue, which is available at wileyonlinelibrary.com.]

skewed toward detection and measurement of so-called "transport pores" which traverse the pellet, and neglect the dead-end pores that still may contain active sites and be important in reactions. However, many transient methods are only one-dimensional (1-D) and supply a single transport coefficient characteristic of the composite behavior of a whole pellet or sample of pellets. In contrast, imaging methods can give spatially resolved, often three-dimensional, information on mass transport.

Imaging methods used to study mass transport in pellets include computerized x-ray tomography ${ }^{10}$ and magnetic resonance imaging (MRI). ${ }^{9}$ However, x-ray imaging is restricted to imaging species containing heavy elements with high electron density, and is limited to density contrast. MRI offers the potential of other contrast methods which can distinguish level of confinement, for example. In the past, MRI has only studied transient diffusion of liquids, or very high pressure gases, because of issues with obtaining sufficient signal-to-noise limiting data to the molecular diffusion regime and to particular chemical species. ${ }^{11}$ More recently, hyperpolarized (hp) gases have enabled PFG NMR ${ }^{12-14}$ and MRI methods to be used to obtain spatially resolved and temporally resolved information on the transport of low pressure gases in porous materials. ${ }^{15-17}$ Gas flow in aerogel silica has been studied ${ }^{18}$ and characterization of microfluidic reactors with para-hydrogen induced polarization $^{19}$ has been performed using remote-detection, or time-of-flight MRI methods using various hp gas species. In Ref. 18, the spatial heterogeneity in the time-of-flight dispersion of hp Xe-129 was imaged. The time-of-flight methodology enables remote detection of events that take place within a reactor that cannot be imaged directly. The transport information for each given region of a sample is convoluted with the transport properties of regions "downstream" of that region of interest (ROI) isolated by the imaging process. However, this makes results sensitive to the subsequent flux pathway, and only indirect data on the mass transport properties of a given zone of the porous medium can be obtained. To serve the purpose of this study, the mass transport properties of each region of the sample will be measured directly to avoid such complications.

In this work, the transient diffusional ingress of hp Xe-129 into the pores of a packed bed of commercial alumina catalyst supports will be studied using MRI. To our knowledge, this is the first quantitative study of transient diffusion for microscopic porous systems using xenon chemical shift to selectively image gas within the pores. This will enable the gas transport heterogeneity across the pellets and the bed to be observed and measured directly and noninvasively. The approach is particularly sensitive to intrabatch variability in commercial pellets. In particular, the method permits the study of pellet heterogeneity on diffusion in the Knudsen regime at relatively low fluid density.

\section{Theory}

For mass-uptake experiments, the appropriate solution to the diffusion equation can be approximated by the so-called linear driving force (LDF) model and uptake data fitted to the expression $^{20}$

$$
\frac{M(t)}{M(\infty)}=1-\exp (-k t)
$$

where $k$ is a mass-transfer coefficient (MTC), equivalent to $8 D_{\mathrm{e}} / r^{2}$, where $r$ is the pellet cylinder radius and $D_{\mathrm{e}}$ is the effective diffusivity. The effective diffusivity is equal to $\varepsilon D_{A} / \tau_{\mathrm{p}}$, where $\varepsilon$ is the voidage fraction, and $\tau_{\mathrm{p}}$ is the pore tortuosity. If the MRI intensity, $I$, is proportional to mass uptake then Eq. 1 implies that 


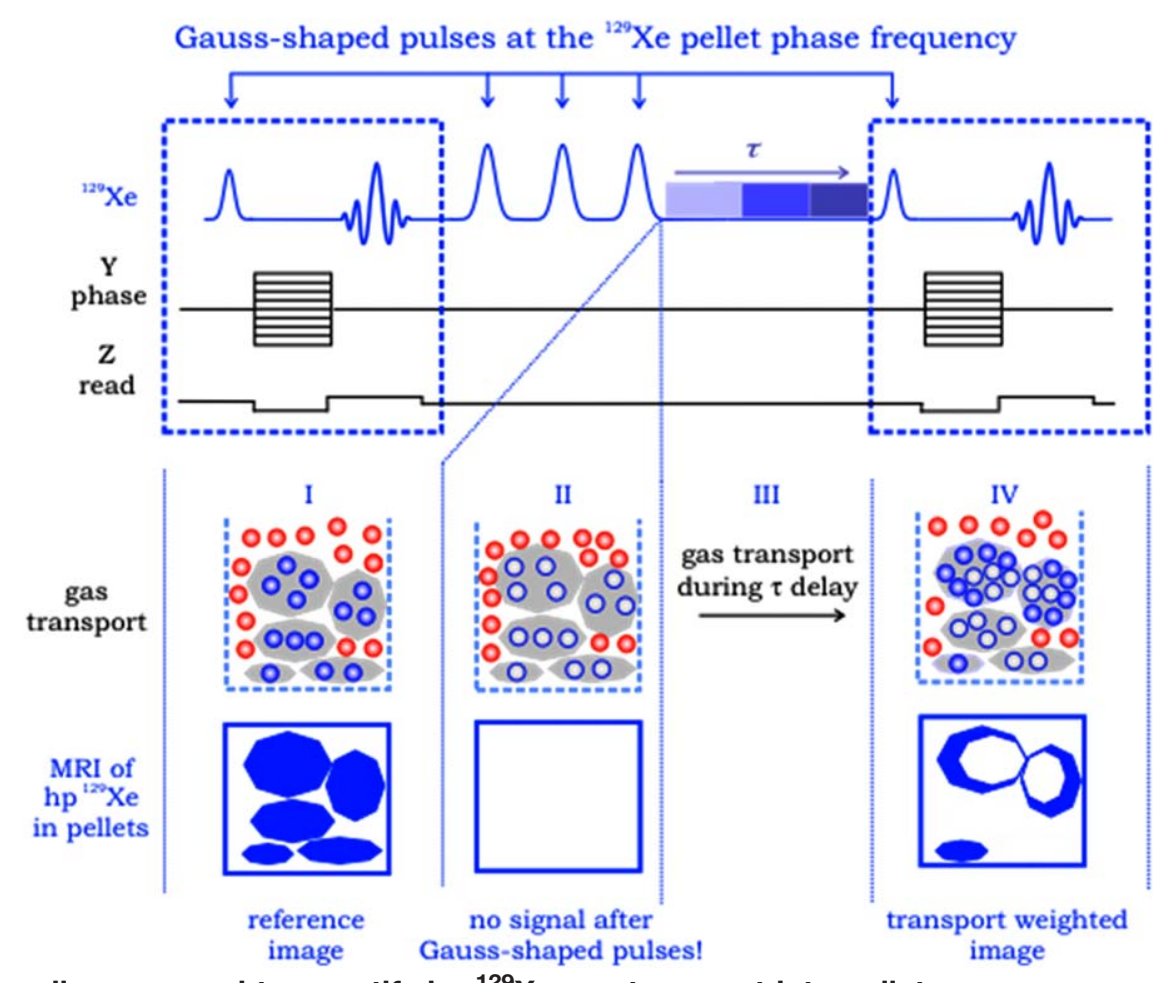

Figure 2. MRI timing diagram used to quantify hp ${ }^{129} \mathrm{Xe}$ gas transport into pellets.

The corresponding gas transport cartoons explain $\mathrm{hp}{ }^{129} \mathrm{Xe}$ behavior in the sample holder during MRI experiment. hp ${ }^{129} \mathrm{Xe}$ in the bulk gas phase is depicted as red filled circles, while hp ${ }^{129} \mathrm{Xe}$ and ${ }^{129} \mathrm{Xe}$ gas in the imbibed (pore) phase are shown as blue filled and blue opened circles, respectively. ${ }^{129} \mathrm{Xe}$ signal is acquired at the xenon resonance frequency of the imbibed phase, however, only hp xenon results in an observable signal. The reference image (Stage I) is acquired right after pressure equalization in the sample holder and shows hp xenon distribution in the pellet phase. This image is also used for the subsequent signal calibration. Three Gaussian-shaped pulses with duration of $900 \mu$ s and $\frac{\pi}{2}$ flip angle separated by a 5-ms delay are applied at the resonance frequency of hp ${ }^{129} \mathrm{Xe}$ in the pellet phase during Stage II. This action ensures that no hp ${ }^{129} \mathrm{Xe}$ signal is detected from the pellet phase resulting in a null image at the end of Stage II. The replacement of the $\mathrm{hp}{ }^{129} \mathrm{Xe}$ through exchange via transport into the pellets occurs during a time delay $\tau$ ( Stage III). The transport-weighted image is recorded after a delay $\tau$ during Stage IV, where hp gas distribution in the pellets is sketched in the cartoon. Both, the reference and transport-weighted images (image loops are highlighted in dashed rectangles in the timing diagram) are acquired using VFA Gaussian pulses. One hp ${ }^{129}$ Xe gas delivery provided enough polarized xenon to record two images (i.e., the reference image and one after a particular delay time $\tau$ ). [Color figure can be viewed in the online issue, which is available at wileyonlinelibrary.com.]

$$
\ln \left(\frac{I(\infty)-I(t)}{I(\infty)}\right)=-k t
$$

Hence, a plot of $\ln [(I(\infty)-I(t)) / I(\infty)]$ for any voxel against preset delay time, $t$, should be a straight line with zero origin and a gradient equal to the MTC.

\section{Experimental \\ Materials}

The material studied in this work, denoted E3, was a commercial cylindrical alumina tablet of diameter $\sim 3 \mathrm{~mm}$ and height $\sim 3 \mathrm{~mm}$ (Engelhardt AL3984T). The BET surface area was $109 \mathrm{~m}^{2} \mathrm{~g}^{-1}$, the specific pore volume was $0.43 \mathrm{~mL} \mathrm{~g}^{-1}$, and the voidage fraction was 0.64 . Mercury porosimetry data analyzed using the modified Kloubek correlations suggested a bimodal pore structure with modal pore sizes of $10 \mathrm{~nm}$ and 0.5 $\mu \mathrm{m} .^{21}$

\section{Hp ${ }^{129} \mathrm{Xe}$ gas production and delivery}

Hp ${ }^{129} \mathrm{Xe}$ was produced through spin exchange optical pumping (SEOP) using a custom-built instrument described elsewhere. ${ }^{22}$ Isotopicaly enriched ${ }^{129} \mathrm{Xe}$ (enriched to $83 \%$ of

${ }^{129} \mathrm{Xe}$ ) was used to prepare a $25 \% \mathrm{Xe}: 75 \% \mathrm{~N}_{2}$ SEOP gas mixture for all experiments in the present work. The SEOP pressure was about $200 \mathrm{kPa}$, and the gas mixture was shuttled into the detection cell via pressure equilibration resulting in a pressure of $159 \mathrm{kPa}$ in the detection cell. The nuclear spin polarization of hp ${ }^{129} \mathrm{Xe}$ was approximately $30 \%$, hence the apparent polarization, ${ }^{22}$ that takes xenon dilution within the mixture into account, was $P_{\text {app }}=7.5 \%$.

In total, eight pellets were used for spectroscopic and imaging experiments. The pellets were placed in a sample holder as shown in Figure 1a. The sample holder was evacuated before each hp gas delivery. The hp gas was then delivered into the sample holder through the valve as shown in the schematic diagram. Imaging sequence or spectroscopic experiments were initiated immediately after pressure stabilization in the sample holder, and pressure was recorded by an in-line pressure gauge. All experiments were conducted at $283 \mathrm{~K}$ and $159 \mathrm{kPa}$ pressure in the container.

\section{NMR and MRI experiments}

A 9.4T Bruker Avance III Microimaging System (Bruker, Germany) and a home built $15-\mathrm{mm}$ saddle coil tuned to ${ }^{129} \mathrm{Xe}$ resonance frequency of $110.69 \mathrm{MHz}$ were used in all NMR and MRI experiments. For excitation of the entire ${ }^{129} \mathrm{Xe}$ spectrum, a rectangular $\frac{\pi}{2}$ radiofrequency pulse with $89 \mu$ s duration was utilized, while chemical shift selective excitation was accomplished through a Gaussian-shaped $\frac{\pi}{2}$ pulse with a width 
(a)

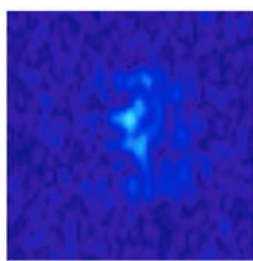

$0.02 \mathrm{~s}$

(b)

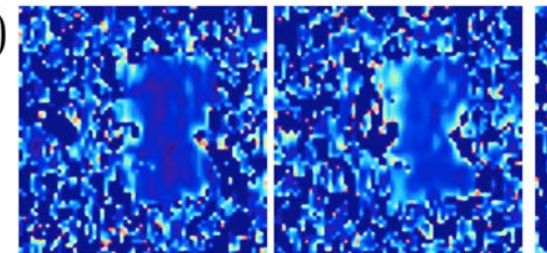

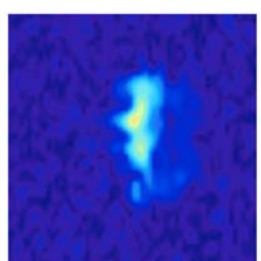

$1.02 \mathrm{~s}$

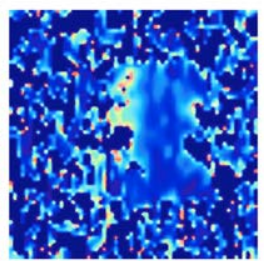

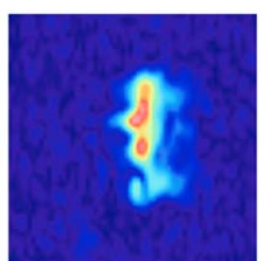

$5.02 \mathrm{~s}$

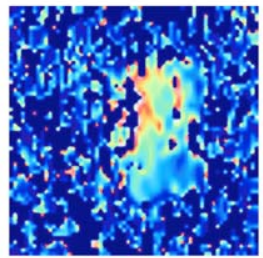

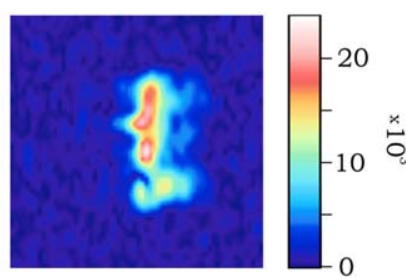

$10.02 \mathrm{~s}$

\section{$\rho_{\omega}$}

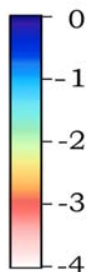

Figure 3. Transport weighted images (a) are shown as function of transport time, $\tau$. The $\tau$ values used in the present work are also shown. Please note that at the pressure of $159 \mathrm{kPa}$, the maximum transport time $\tau=10.02 \mathrm{~s}$ was used in order to minimize $T_{1}$ relaxation effects of xenon. Images used for transport coefficient calculations are shown in (b). This set of images was produced from images shown in (a) by subtracting each $\tau$ image from $\tau=10.02 \mathrm{~s}$ image. This procedure allowed for the determination of transport coefficients from linear fitting according to Eq. 2.

[Color figure can be viewed in the online issue, which is available at wileyonlinelibrary.com.]

of $900 \mu \mathrm{s}$. A nonselective, single pulse sequence was used for 1-D spectroscopy. The spectral width was adjusted to observe

${ }^{129} \mathrm{Xe}$ signal originating from both the bulk gas-phase xenon and the xenon gas imbibed in the pellet phase. Image acquisition was obtained through a variable flip angle (VFA) gradient echo scheme. ${ }^{23}$ The MR images were collected either at the resonance frequency of the bulk gas phase (referenced to 0 ppm, see Figure $1 \mathrm{~b}$ ), or that of the pellet phase (i.e., at 58 ppm), and were acquired into $64 \times 32$ data matrices with field of view $=29.4 \times 21.5 \mathrm{~mm}^{2}$ as shown in Figure 1a. Image parameters were kept the same for all MRI experiments reported in this study.

The timing diagram of the MRI protocol developed specifically for transport-weighted studies in porous solids is displayed in Figure 2. Note that the reference and transportweighted images were acquired with chemical shift selection at the ${ }^{129} \mathrm{Xe}$ resonance frequency originating from xenon within the porous pellets (i.e., at $58 \mathrm{ppm}$ - see Figure 1b). The reference image is recorded immediately after pressure equalization in the sample chamber. Following the reference image, a train of three high power Gaussian pulses is applied at the 58 ppm chemical shift offset from the gas-phase xenon resonance in order to destroy the ${ }^{129} \mathrm{Xe}$ hyperpolarization within the pellet phase. This causes the pellets to appear dark in a second MR image recorded immediately after the Gaussian pulse train, as no hp ${ }^{129} \mathrm{Xe}$ signal can be obtained from the pellet phase at this point. However, the spin polarization of bulk gas phase xenon is left intact and over time hp ${ }^{129} \mathrm{Xe}$ from the bulk gas phase will penetrate into pellets. The (incremented) time period $\tau$ time in the pulse sequence in Figure 2 allows for this diffusion process to happen. Therefore, if the MR images are acquired after a nonzero $\tau$ interval, bright areas appear in the MR image because hp ${ }^{129} \mathrm{Xe}$ entered the pellet phase during the $\tau$ time delay. These transport-weighted MRI experiments are repeated for several $\tau$ values, thus monitoring various degrees of diffusion of the gas-phase hp ${ }^{129} \mathrm{Xe}$ into the pellets. Note that for each hp ${ }^{129} \mathrm{Xe}$ gas delivery one reference and one transport weighted image (following one particular $\tau$ delay time) are recorded.

In principle, the train of three Gaussian pulses applied immediately after the reference image is recorded is sufficient to destroy any significant magnetization of the hp xenon in the pore space. The signal from the hp xenon in the pellet phase decays to $\sim 1 / e$ of the original value in $\sim 420 \mu \mathrm{s}$, and the total length of the delay period following each Gaussian pulse in the train is $\sim 5 \mathrm{~ms}$. Hence, this time represents $\sim 12$ characteristic time periods for the signal decay process, and would mean the signal decayed to $\sim(1 / e)^{12}$ of the original value, effectively returning the nuclear spin polarization to values of the thermal polarization that will not produce a detectable MRI signal. The second and third pulses are to ensure total signal decay even if the pulses are not perfect $\frac{\pi}{2}$ excitation pulses. Furthermore, diffusion in the magnetic field gradient within the pores during the delay time will largely prevent refocusing of dephased xenon signal by the second or third pulse.

\section{Data processing and analysis}

Data were processed using Prospa (Magritek, New Zealand). Raw $64 \times 32$ data matrices were apodized using sincsquared window function and zero filled to 64 in the phase encoding dimension before image reconstruction using twodimensional Fourier transformation. The spatial resolution in the resulting images was $0.459 \times 0.335 \mathrm{~mm}^{2}$. Subsequent mass transport data analysis with bimodal Gaussian fitting was done with IgorPro 6.2 (Wavemetrics) using build-in MultiPeak fitting 1.4 package.

\section{Results}

Xenon gas imbibed in the pellets shows a distinct resonance located at $58 \mathrm{ppm}$ downfield from the bulk phase gas signal (at 


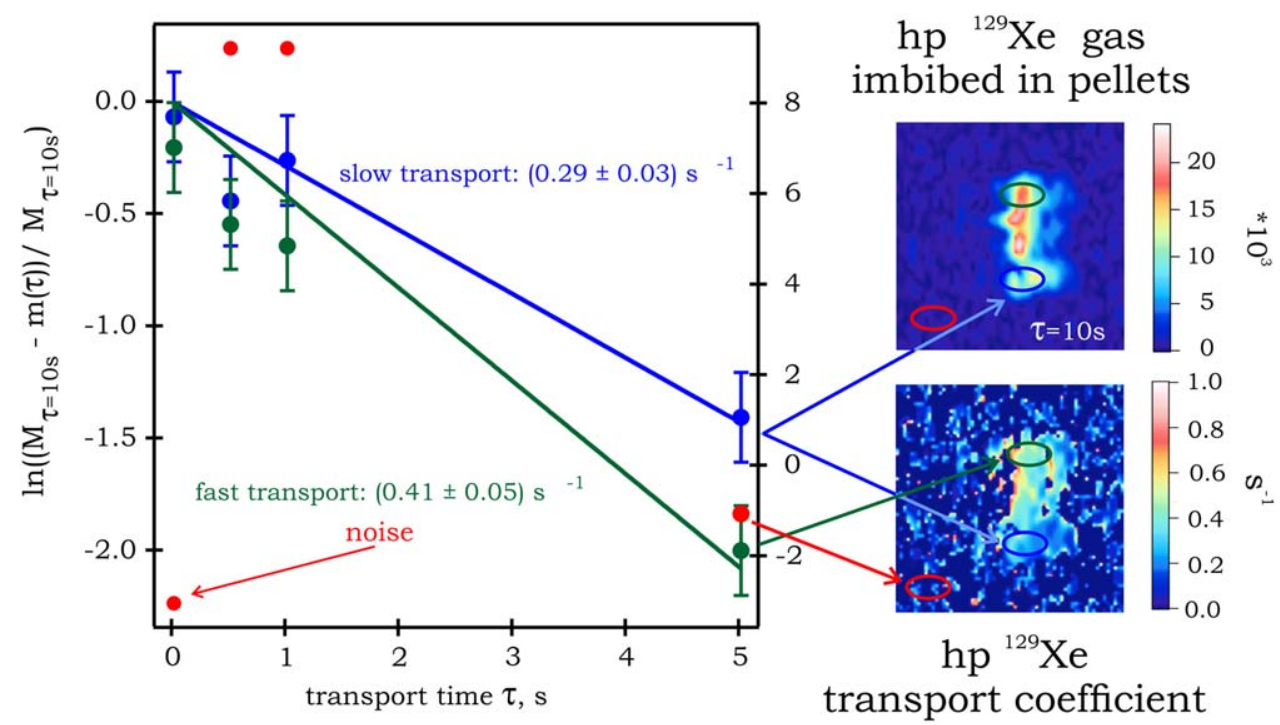

Figure 4. Linear fitting analysis of $\mathrm{hp}{ }^{129} \mathrm{Xe}$ transport in pellets using transport weighted images displayed in Figure $3 b$.

The sample data collected from a voxel within the color encoded area as shown in $\tau=10.02 \mathrm{~s}$ image displayed in the upper right panel. Representative noise data collected from the area outside the ROI are also shown. Please note that only the data collected from the ROI demonstrate meaningful linear dependence $(R>0.92)$ of $\ln \left(\left(M_{\tau}=10 \mathrm{~s}-m(\tau)\right) / M_{\tau}=10 \mathrm{~s}\right)$ quantity with the increase of transport time $\tau$ as shown in the left panel. The gradients resulted from the fitting demonstrate heterogeneity in transport properties in the pellets from the same batch. This heterogeneity is highlighted in the two-dimensional map of hp ${ }^{129}$ Xe gas transport coefficients in the pellets as shown in the lower right panel. [Color figure can be viewed in the online issue, which is available at wileyonlinelibrary.com.]

$0 \mathrm{ppm}$ ) as shown in the $1-\mathrm{D}{ }^{129} \mathrm{Xe}$ NMR spectrum after loading the pellets with the hp gas (Figure 1b). It is possible to produce an image of xenon gas located in either phase if the frequency offset in the imaging experiment is matched to the ${ }^{129} \mathrm{Xe}$ frequency in the corresponding phase. Hp Xe-129 images of the free xenon gas and the xenon imbibed in the pellets produced using this technique are displayed in Figure 1a. The free gas image $(0 \mathrm{ppm})$ shows the xenon distribution in the sample holder with the gas-phase space above the pellets appearing as a bright region. To a lesser extent, gas-phase ${ }^{129} \mathrm{Xe}$ is detected in the pellet region, originating from the void spaces between the pellets. Indeed, the MRI of the free gas reveals the voidage variation in the packed bed of pellets. Conversely, the image acquired at the pellet resonance frequency (58 ppm) captures xenon located predominantly in the pellet phase with the absence of any xenon signal outside the pellet area. The composite image produced by combining the gas-phase and the pellet phase xenon images is displayed in Figure $1 \mathrm{~b}$. The composite image demonstrates that both images are complementary and reflect spatial distribution of xenon gas in the whole sample holder. Moreover, both images match well at the boundary. The ability to distinguish ${ }^{129} \mathrm{Xe}$ in the free gas phase from the adsorbed phase within the pellet through different MRI frequencies allows for unique transport weighted MRI experiments.

Transport-weighted images (at $159 \mathrm{kPa}, 283 \mathrm{~K}$ ) are displayed in Figure 3a. The sample was pre-evacuated and the hp gas was then delivered. The reference image of xenon imbibed in the pellets acquired right after the pressure equalization in the sample chamber was used for the signal intensity calibration. The transport-weighted image was recorded after destroying any residual xenon in the pellet phase and waiting for gas-phase $\mathrm{hp}{ }^{129} \mathrm{Xe}$ to penetrate the pellets via diffusion during the period, $\tau$. With increasing $\tau$ period, an increasing number of ${ }^{129} \mathrm{Xe}$ atoms were able to enter the pellets and lead to increased signal detected in the pellet phase. The maximum duration for this process was limited to $\tau=10.02 \mathrm{~s}$ to minimize competing effects of the ${ }^{129} \mathrm{Xe} T_{1}$ relaxation. The $T_{1}$ relaxation leads to a depolarization of $\mathrm{hp}{ }^{129} \mathrm{Xe}$ and hence to a signal decay that for longer $\tau$ times needs to be taken into account to avoid incorrect interpretation of gas transport behavior. The $T_{1}$ in the pellet phase is faster than in the gas phase but may also vary within the pellets but $T_{1}$ is typically many tens of seconds in this type of material. To apply Eq. 2, the data were rearranged by subtracting each image from the image at $\tau=10.02 \mathrm{~s}$, dividing the sum by the image at $\tau=$ $10.02 \mathrm{~s}$ and applying natural logarithm to the data resulting in the images shown in Figure 3b. These images were used for subsequent mass transport coefficient (MTC) calculations. The data extracted from a given voxel were plotted as a function of $\tau$ time as shown in the left panel of Figure 4. The data were collected from different spatial regions in the pellet bed reflecting areas of slower and faster gas uptake as shown in the upper right panel of Figure 4, (the $\tau=10.02 \mathrm{~s}$ was used as the gas uptake reference). The data were analyzed using Eq. 2 to obtain the MTC value of the gas in each voxel. The MTC values were obtained from one parameter linear fits as shown in Figure 4. The correlation coefficients for these lines were above 0.92 for all the data analyzed. The resulting transport coefficient map is displayed in the lower right panel in Figure 4. Please note that the data collected outside the ROI (shown in red) showed no linear dependence.

The gas uptake intensity map at $\tau=10.02$ (Figure 5a) was used to define the ROI in order to reject any noise values located outside the ROI boundary. All statistically significant data were used to produce the distribution of MTCs in pellets. The data in the interval of $\left(0-1.5 \mathrm{~s}^{-1}\right)$ were collected from the inside of the ROI boundary in the image displayed in Figure 5a and binned with the resolution of $0.01 \mathrm{~s}^{-1}$. The resulting histogram of MTCs in the pellets is displayed in Figure 5b. The 

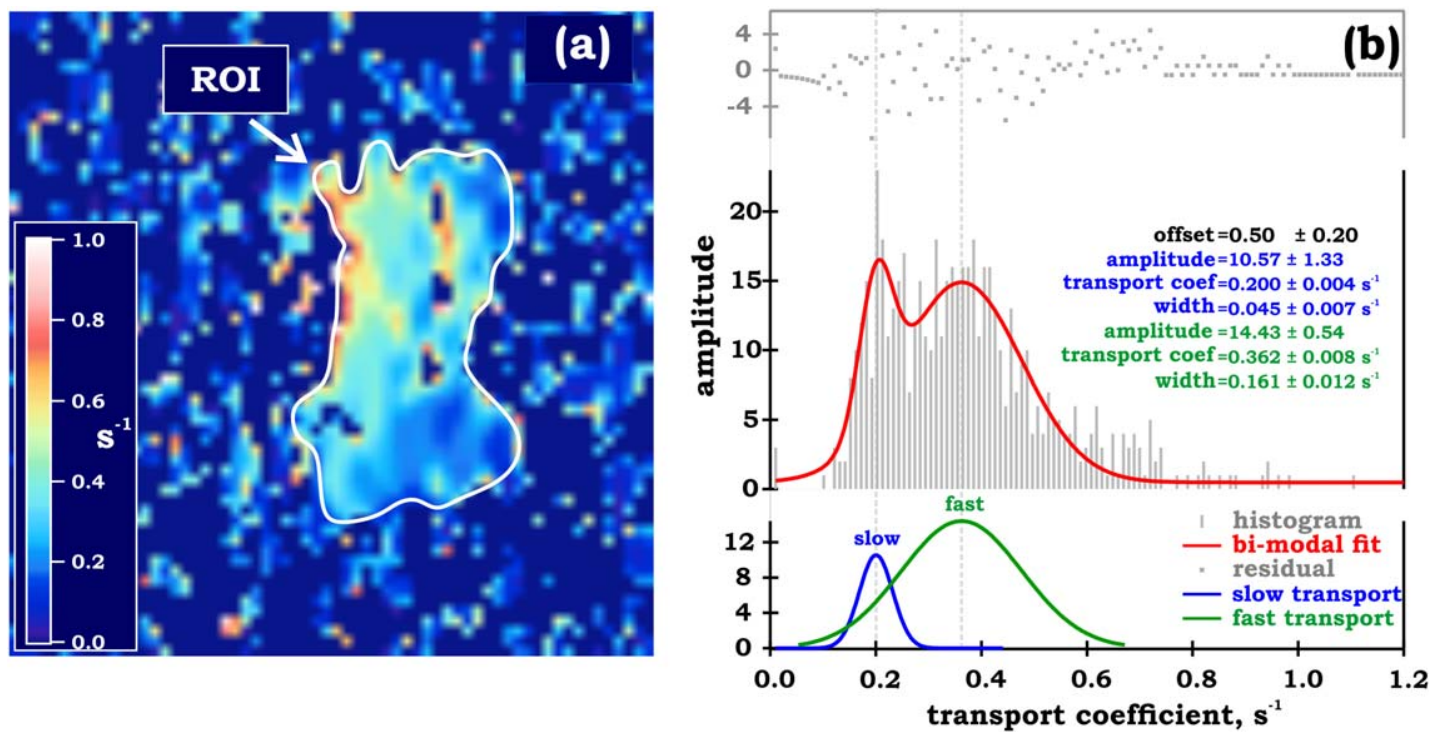

Figure 5. (a) The two-dimensional map of $\mathrm{hp}{ }^{129} \mathrm{Xe}$ gas transport in the pellets highlighting spatial heterogeneity of this process. (b) The distribution of xenon gas transport coefficients in the pellets.

Two major modes are observed hence bi-modal Gaussian distribution of mass transport coefficients was assumed. The modes of this complex process are displayed in blue and green after bimodal Gaussian analysis. The fitting parameters of deconvoluted modes are shown in the inset with corresponding colors. Please note that the determined mean transport coefficients coincide well with the prediction. [Color figure can be viewed in the online issue, which is available at wileyonlinelibrary.com.]

distribution appears to be multimodal but was deconvoluted using bimodal Gaussian distribution as only the major modes were considered to be significant within experimental variations. The parameters of deconvoluted modes are also reported in Figure 5b, and are discussed in the next section. The ratio of the standard deviation to the mean, known as the reduced standard deviation, for the distribution of MTCs is 0.44 .

While this polarization destruction period is very short relative to the characteristic time-scale of mass transport into the pellets, it is conceivable that some hp xenon might diffuse into the material during the third Gaussian pulse leading to nonzero signal intensity immediately after the depolarization sequence. To estimate the effect of this process on our measurements, we fitted an alternative version of the standard LDF model that included a boundary condition where the starting concentration was not zero. It was found that this model made no difference to the form of the fitted distribution (bimodal), nor the spread in mass-transfer coefficients obtained.

\section{Discussion}

The conditions of temperature and pressure of the experiment are such that the mean free path (calculated from kinetic theory) is $\sim 30 \mathrm{~nm}$. This is larger than the modal mesopore size, but smaller than the macropore size, for the alumina tablets. Hence, diffusion would be in the Knudsen regime in the mesopores but in the molecular regime in the macropores. This is commonly the situation for bidisperse pellets during industrial operation of many processes.

The well-known model of Wakao and $\mathrm{Smith}^{24}$ for bidisperse systems was used to predict a value of $k \tau_{\mathrm{p}}$ and was found to be $1.2 \mathrm{~s}^{-1}$. If the pore-scale tortuosity $\tau_{\mathrm{p}}$ was similar to that found ${ }^{5}$ for PFG NMR of $\sim 2$, then $k \sim 0.6 \mathrm{~s}^{-1}$, which is just in the range of measured values, as the error is about $10 \%$. However, the slightly lower overall mean value of $k$ measured using gas-phase MRI of $\sim 0.36 \mathrm{~s}^{-1}$ may reflect that there is additional tortuosity over length-scales larger than up to $\sim 10$ s microns probed by liquid-state PFG NMR caused by the larger scale heterogeneity as seen in Figure 4.

As mentioned above, knowledge of the bed heterogeneity in diffusion is necessary for predicting reactor performance on activity and selectivity. The variation in the MTC across the packed bed, as seen in Figures 4 and 5 suggests significant heterogeneity to gas-phase diffusion between pellets from the same batch. When characterizing the diffusional heterogeneity in small samples of porous pellets techniques based on molecular diffusion, such as PFG NMR of imbibed liquids, are often employed. ${ }^{5}$ In critical path analysis of mass transport, ${ }^{25}$ for molecular diffusion, the pore conductance is proportional to $r^{2}$, while in Knudsen diffusion conductance is proportional to $r^{3}$. Due to the higher power dependence on $r$, the reduced standard deviation in the Knudsen diffusivity should be larger than for molecular diffusivity. However, the difference will depend upon the nature of the pore space. The lower gasphase MTC may also reflect the additional tortuosity, arising from the distribution in resistances for Knudsen diffusion due to the pore-size variation within the pellets, that would not be evident for molecular diffusion studied using liquid-state PFG NMR. It has also been found that the diffusional uptake of xenon gas in the packed bed of bimodal macroporous/mesoporous alumina tablets had a bimodal distribution in MTC, with an overall reduced standard deviation of 0.44 . Previous work ${ }^{5}$ that used PFG NMR to measure the distribution of bulk diffusivity divided by tortuosity in pellets of E3 found a reduced standard deviation of $\sim 0.3$ for a two-component distribution and $\sim 0.4$ for a uniform distribution. Hence, the spread in conductances was at least $10 \%$ wider for Knudsen diffusion than for molecular diffusion.

\section{Conclusions}

Gas-phase MRI has been shown to be a useful technique for characterizing transport heterogeneity in a packed bed of porous catalyst support pellets. The mechanistic regime for 
diffusion in the hp xenon experiment is closer to that for typical industrial processes than in other types of NMR and MRI experiment that are limited to molecular diffusion because of the need for high probe fluid density. The new experiments demonstrate that variability in pellet performance across the bed is likely due to the observed heterogeneity in mass transport.

\section{Acknowledgment}

This work was supported in part through the Royal Society through the Paul Instrument Fund.

\section{Literature Cited}

1. Westerterp KR, Swaaij WPMv, Beenackers AACM. Chemical Reactor Design and Operation. London: Wiley, 1984.

2. Beeckman JW, Froment GF. Catalyst deactivation by active site coverage and pore blockage. Ind Eng Chem Fundam. 1979;18:245-256.

3. Rigby SP, Beanlands K, Evbuomwan IO, Watt-Smith MJ, Edler KJ, Fletcher RS. Nanocasting of novel, designer-structured catalyst supports. Chem Eng Sci. 2004;59:5113-5120.

4. Capek P, Vesely M, Hejtmanek V. On the measurement of transport parameters of porous solids in permeation and Wicke-Kallenbach cells. Chem Eng Sci. 2014;118:192-207.

5. Hollewand MP, Gladden LF. Transport heterogeneity in porous pellets.1. PGSE NMR-studies. Chem Eng Sci. 1995;50:309-326.

6. Watt-Smith MJ, Kolaczkowski ST, Rigby SP, Chudek JA. Prediction of gas sorption kinetics for porous media using MRI. AIChE J. 2006;52:3278-3289.

7. Calvo-Almazan I, Bahn E, Koza MM, Zbiri M, Maccarini M, Telling MTF, Miret-Artes S, Fouquet P. Benzene diffusion on graphite described by a rough hard disk model. Carbon. 2014;79:183-191.

8. Cresswell DL, Orr NH. Residence Time Distribution Theory in Chemical Engineering. Weinheim: Verlag-Chemie, 1982:41-74.

9. Hollewand MP, Gladden LF. Transport heterogeneity in porous pellets. 2. NMR imaging studies under transient and steady-state conditions. Chem Eng Sci. 1995;50:327-344.

10. Koestel J, Larsbo M. Imaging and quantification of preferential solute transport in soil macropores. Water Resour Res. 2014;50: $4357-4378$.

11. Koptyug IV. MRI of mass transport in porous media: drying and sorption processes. Prog Nucl Magn Reson Spectrosc. 2012;65:1-65.
12. Mair RW, Wong GP, Hoffmann D, Hurlimann MD, Patz S, Schwartz LM, Walsworth RL. Probing porous media with gas diffusion NMR. Phys Rev Lett. 1999;83:3324-3327.

13. Mair RW, Sen MN, Hurlimann MD, Patz S, Cory DG, Walsworth $\mathrm{RL}$. The narrow pulse approximation and long length scale determination in xenon gas diffusion NMR studies of model porous media. J Magn Reson. 2002;156:202-212.

14. Mair RW, Wang R, Rosen MS, Candela D, Cory DG, Walsworth RL. Applications of controlled-flow laser-polarized xenon gas to porous and granular media study. Magn Reson Imaging. 2003;21: 287-292.

15. Kaiser LG, Meersmann T, Logan JW, Pines A. Visualization of gas flow and diffusion in porous media. Proc Natl Acad Sci USA. 2000; 97:2414-2418.

16. Kaiser LG, Logan JW, Meersmann T, Pines A. Dynamic NMR microscopy of gas phase Poiseuille flow. J Magn Reson. 2001;149: $144-148$.

17. Kovtunov KV, Zhivonitko VV, Skovpin IV, Barskiy DA, Koptyug IV. Parahydrogen-induced polarization in heterogeneous catalytic processes. Top Curr Chem. 2013;338:123-180.

18. Harel E, Granwehr J, Seeley JA, Pines A. Multiphase imaging of gas flow in a nanoporous material using remote-detection NMR. Nat Mater. 2006;5:321-327.

19. Zhivonitko VV, Telkki VV, Koptyug IV. Characterization of microfluidic gas reactors using remote-detection MRI and parahydrogeninduced polarization. Angew Chem Int Ed. 2012;51:8054-8058.

20. Do D. Adsorption Analysis: Equilibria and Kinetics. London: Imperial College Press, 1998.

21. Rigby SP, Fletcher RS. Interfacing mercury porosimetry with nitrogen sorption. Part Part Syst Charact. 2004;21:138-148.

22. Six JS, Hughes-Riley T, Stupic KF, Pavlovskaya GE, Meersmann T. Pathway to cryogen free production of hyperpolarized Krypton-83 and Xenon-129. PLOS One. 2012;7:e49927.

23. Zhao L, Mulkern R, Tseng CH, Williamson D, Patz S, Kraft R, Walsworth RL, Jolesz FA, Albert MS. Gradient-echo imaging considerations for hyperpolarized Xe-129 MR. J Magn Reson Ser B. 1996;113:179-183.

24. Wakao N, Smith JM. Diffusion and reaction in porous catalysts. Ind Eng Chem Fundam. 1964;3:123-127.

25. Friedman SP, Seaton NA. Critical path analysis of the relationship between permeability and electrical conductivity of threedimensional pore networks. Water Resour Res. 1998;34:1703-1710.

Manuscript received Feb. 20, 2015, and revision received May 15, 2015. 